\title{
A lithological and morphological map created from multibeam backscatter data in challenging circumstances: the Lower Sea Scheldt estuary
}

Mieke MATHYS, Belgium

International Marine and Dredging Consultants (IMDC), Coveliersstraat 15, B-2600 Berchem, Belgium

- Email: mim@imdc.be. T: +32 327092 21. www.imdc.be

Marc SAS, Belgium

International Marine and Dredging Consultants (IMDC), Coveliersstraat 15, B-2600 Berchem, Belgium

- Email: msa@imdc.be. T: +32 327092 21. www.imdc.be

Frederik ROOSE, Belgium

Flemish Ministry of Mobility and Public Works, Maritime Access Division, Tavernierkaai 3, B2000

Antwerp, Belgium - Email: Frederik.Roose@mow.vlaanderen.be and. T: +32 32220818.

www.maritiemetoegang.be

Topic H: geophysics of the marine environment

\section{INTRODUCTION}

The Lower Sea Scheldt is regularly dredged to maintain the access to the harbour of Antwerp. The Maritime Access Division has the permission to relocate the dredged material within the Scheldt itself on the condition that a monitoring program is performed in order to detect any negative effects on the environment. The creation of a lithological and morphological map of the entire Lower Sea Scheldt is part of an extensive integrated monitoring program.

In the past, a lithological map was created based on the backscatter signal from singlebeam profiles and the morphological maps were created based on side-scan sonar data. The presented study investigated the feasibility and applied the most appropriate method to create a lithological and morphological map from full coverage multibeam backscatter data which are anyhow available from the regular depth soundings in the Lower Sea Scheldt.

\section{METHODS}

Creation of a lithological map

For the creation of the lithological map QTC MULTIVIEW ${ }^{\mathrm{TM}}$ is used. It is an integrated software package that classifies sediments using the amplitudes and statistical properties of backscatter images. Information is extracted from the amplitudes and variability of backscatter, and detailed bathymetry from the same survey is used for geometrical image compensation. Classification of the river bottom is done by an automated clustering method that adapts to the characteristics of the multibeam set. Each cluster or acoustic class represents a bottom type that can be identified based on ground truth.

If the bottom type is known before classification, data from the areas of diverse sediment types can be used to build a catalogue, which could then be used to classify subsequent or archived data. This is called supervised classification. The alternative, unsupervised classification, forms the data into logical clusters that can then be identified based on ground truth.

Creation of a morphological map

In the past, morphological maps were created based on side-scan sonar images. The disadvantage of this technique is that certain reflections due to sedimentological characteristics of the river bottom could not be distinguished from reflections due to the morphological character of the area. 
A test was performed comparing the performance of both side-scan sonar and multibeam sonar over the same areas.

The following seven structural elements were recognized: large dunes $(\mathrm{H}=75 \mathrm{~cm}-5 \mathrm{~m} ; \lambda=10 \mathrm{~m}-$ $100 \mathrm{~m})$, small to middle-large dunes $(\mathrm{H}=7,5 \mathrm{~cm}-75 \mathrm{~cm} ; \lambda=60 \mathrm{~cm}-10 \mathrm{~m})$, slides, small scale cliffs, structureless bottom, dredging traces, irregular bottom structures.

\section{Multibeam campaigns}

Two different ships (Veremans and Parel2) were used to cover the Lower Sea Scheldt. One ship was used to sound the shallow tidal flat areas, the other ship recorded the deep channel areas. Multibeam sonars of type Simrad EM 3002 dual (frequency $300 \mathrm{kHz}$, pulse rate $10 \mathrm{~Hz}$, pulse length 149 us) were applied. Six section maps were created.

Sampling campaigns and lab tests

Shortly after the multibeam recordings, for each section map, fifty control samples were taken with a Shipek bottom sampler. The samples were described and photographed on board and later analysed with a (laser diffraction) Malvern Mastersizer.

With the purpose of performing a supervised classification, at six reference locations, Shipek, Van Veen and box core samples were taken, during flood, ebb and slack time, followed by multibeam measurements in each area. The six reference locations represented areas with different lithology: sand, sandy mud, muddy sand, mud, hard soil due to clay, hard soil due to gravel. The backscatter data of the six reference zones were processed together to obtain a catalogue. Grain-size, carbon content and density profiles were determined of each calibration sample. To visualise the vertical structure of the subsurface also radiographs were taken.

In a final stage, validation samples were acquired to check the validity of the lithological map in areas which were not sampled before.

\section{CHALLENGING CIRCUMSTANCES}

The Lower Sea Scheldt estuary confronts us with challenging circumstances which are not present when mapping offshore areas with multibeam: during a tidal cycle, sediment concentration, salinity and water depth changes significantly. Moreover, due to the morphological character of the Lower Sea Scheldt two different ships were put into action to be able to cover both the deep channels and shallow tidal flats with multibeam.

\section{Slack water and influence of sediment concentration}

Slack water is the moment when the ebb current changes into flood current (low water slack time) and reverse (high water slack time). Theoretically this coincides with the moment when the current velocity is zero. The moment of low and high water slack coincides, moreover, with the moment of minimum and maximum salinity respectively. During slack time, the current velocity is that low, that suspended sediment particles settle and form a high concentrated mud layer near the bottom. As a high-frequency $(300 \mathrm{kHz})$ multibeam is used, it is not unrealistic that during slack time, backscatter returns from the settled mud particles instead of the underlying bottom.

Two tests were performed to investigate the variations in acoustic backscatter during slack water. One test ran from high water to 2.5 hours after high water. The second test ran from 0.5 hours before low water to 1.75 hours after low water. During these periods, one track was repeatedly recorded and the created acoustic classification maps were compared. Only little variation was observed in the acoustic backscatter during slack water, mainly at the boundaries of the acoustic classes possibly due to differences in ship positioning between successive tracks. 
Absorption of noise by sea water is an important factor that can influence the results of highfrequency multibeam sonars (depending on temperature, water depth, salinity). This is especially the case in the Scheldt estuary where the salinity and water depth changes strongly over the tidal period and stratification occurs between fresh and salt water.

The QTC MULTIVIEW ${ }^{\mathrm{TM}}$ software is however developed in such a way that regular CTD measurements (Conductivity, Temperature, Depth) are not necessary. It concerns a classification method without knowing the absorption coefficient. An important part of the tool is a patented technique using compensation tables. The QTC compensation results in images consistent throughout the entire survey (normalized over the entire survey). Backscatter is not expressed in physical units (e.g. backscatter amplitude in decibels), but in relative image amplitudes on a scale from 0 to 255 .

Nevertheless, the effect of salinity and the effectiveness of the software was tested using a simple set-up. The same survey line was analysed in case of two extreme salinity values (sound velocities). Few differences were observed between the positions of the footprints of the outermost beams, who would experience the strongest effect due to the longer distance these beams travel through the water column.

Changes in water depth also have an effect on swath width and resolution. In shallower areas, the swath width and foot print are smaller, but the same information is present, so more detail is captured. During the soundings one also has to ensure that the overlap between survey lines is sufficient. With changing water depth over the tide cyle, the spacing between survey lines has to be adapted.

\section{Recordings with two vessels}

The effect of applying two different vessels with two multibeam transducers each, was tested qualitatively and quantitatively. In a first, qualitative test, the classification in acoustic classes was done on data from Parel2 and Veremans separately, and on a combination of the two integrated data sets, after which the resulting classification maps were compared. The integrated classification map differs clearly from the separate maps, mainly at the boundaries of the classes. The differences depend strongly on the percentage of the surface covered by the individual data sets.

In a second, quantitative test, the same two survey lines were recorded by both Parel 2 and Veremans (in the same direction) and the classification maps were subtracted to create difference maps. The differences between recordings of two vessels in an identical area were expressed in $\%$ of the total surface area. Also the reproducibility of a single transducer was tested, by covering a single line three times, over a time span of 8 minutes, in the same direction, with the same ship. Comparisons showed that differences between sequential measurements over a single line with the same transducer, were in the same order of magnitude as differences between transducers of two different ships, both in an acoustic homogeneous and heterogeneous area.

\section{Influence of oblique incidence}

From the previous test set-up, also the influence of the grazing and incidence angle (Figure 1) could be estimated, as a certain area was covered under two different angles with the same ship. A smaller incidence angle corresponds to a shorter distance between source and bottom, so less absorption in the water column. Results showed however that minor differences were due to navigation differences and not due a systematic error. It could be concluded that the QTC MULTIVIEW ${ }^{\mathrm{TM}}$ software compensates correctly for differences in travelled distance of the sound wave. 
The grazing angle depends not only on the beam angle of the transmitted signal, but also on the local relief or morphology. That angle will determine how strong the signal is reflected and/or refracted. Also this effect is compensated by QTC MULTIVIEW ${ }^{\mathrm{TM}}$, and points with too sharp and too perpendicular grazing angles are removed from the data set.

\section{Time variations}

As the section maps of the Lower Sea Scheldt were recorded over a period of more than one month, the effect of the time interval was investigated. Differences in maps created from data recorded with 1 week interval, apppeared to be mainly due to differences in positioning, than due to lithological changes. One exception, but only visible on the bathymetric maps, when sections were sounded just before and just after dredging works, adjacent sections show then a clear different morphology (cutoff dredging tracks next to smooth surface).

\section{CREATION OF A LITHOLOGICAL MAP}

Processing and visualisation of data

In a first step the ideal parameter settings were investigated to processes the raw data (threshold value for removal of spikes, masking of beams with certain grazing angle, removal of overlapping beams). And the best solution was sougth between data reduction (to reduce calculation time) and resolution of the maps.

In a second step, the best visualisation parameters were defined for the final classification maps (grid cell size, interpolation distance, number of data points within interpolation radius). The best settings showed no longer the sailing tracks, but still enough heterogenity in acoustic classes.

Afterwards, the influence of the software clustering settings on the resulting classification maps was tested. Using a different number of records, may result in a different number of acoustic classes. The minimal number of records and iterations needed for the clustering process were determined $(50 \%$ and 5 iteractions resp.).

The number of acoustic classes can be determined by the user (supervised classification) or the optimum number of classes can be determined statistically by the software (within a choosen range) (unsupervised classification).

\section{Supervised classification}

Initially, a supervised classification was performed: the backscatter data of six lithological reference zones were processed together to obtain a 'catalogue' with a number of acoustic classes. Various catalogues with a different preset number of acoustic classes (6-7-9-15 classes) were tested by comparing the obtained classification maps in the reference areas with the calibration sediment samples. The best catalogue would then be applied to the entire Lower Sea Scheldt multibeam data set.

A catalogue of six acoustic classes was aimed for (corresponding to six lithologies: sand, sandy mud, muddy sand, mud, hard soil due to clay, hard soil due to gravel). But in fact, the catalogue which gave the best results (most samples with the same lithology belonging to the same acoustic class, least outliers within a certain class) had 15 acoustic classes. However, after applying this catalogue to the entire Lower Sea Scheldt data set, only 3 lithological facies could be discerned: mud, hard soil and a class covering 'sand-sandy mud-muddy sand'. After comparison with calibration and validation samples, it appeared that more than one acoustic class had the same lithology and that within one acoustic class different lithologies occurred.

Unsupervised classification

As the supervised classification map did not give any surplus value over past maps, also unsupervised classification was considered. A catalogue was created now from the entire Lower Sea Scheldt multibeam data set, instead of using only a restricted reference area as was the case for the 
supervised classification. The optimum number of acoustic classes was statistically determined by the software iteration process, i.e. 14 acoustic classes. Afterwards the catalogue was applied to the same Lower Sea Scheldt data set to get a classification map. Based on the sediment samples, the acoustic classes could be lithologically interpreted.

The unsupervised classification map was considered the best lithological map, discerning 5 lithological classes: sand, hard soil, mud and two classes with different acoustic signature of 'sand-sandy mudmuddy sand'. Most likely the unsupervised map discerns more lithological classes than the supervised map because the complete backscatter dataset was used to set up the catalogue, which is important in a heterogeneous area as the Scheldt estuary.

\section{CREATION OF A MORPHOLOGICAL MAP}

Eight zones with a typical morphology were simultaneously surveyed with side-scan sonar (SSS) and multibeam. Comparison of the data showed that multibeam bathymetric data are more suited to distinguish morphological elements than SSS data. SSS data give a detailed photographic image of the seafloor, but contain no bathymetric information. Morphological structures, such as dune, cliffs and dredging tracks, are identified from differences in backscatter intensity. However, strong backscatter can on the one hand indicate that the seafloor is facing the transducer, but on the other hand, can also indicate a lithological facies like sand. In an area where on multibeam data clear bathymetric differences were recorded, on the SSS data, shade differences were wrongly interpreted as facies differences on a structureless bottom.

To be able to detect morphological units, hillshades were generated from the multibeam data. It is a shadow with a certain azimuth, altitude and exaggeration factor, placed on top of the bathymetric map to visually accentuate bathymetric differences. Seven structural elements were mapped by manually drawing polygons around areas with: large dunes $(H=75 \mathrm{~cm}-5 \mathrm{~m} ; \lambda=10 \mathrm{~m}-100 \mathrm{~m})$, small to middle-large dunes $(H=7,5 \mathrm{~cm}-75 \mathrm{~cm} ; \lambda=60 \mathrm{~cm}-10 \mathrm{~m})$, slides, small scale cliffs, structureless bottom, dredging traces and irregular bottom structures.

\section{RECOMMENDATIONS}

\section{Frequency multibeam}

The applied high-frequency multibeam ( $300 \mathrm{kHz})$ gives a high resolution, but a very shallow penetration depth. The backscatter signal is therefore only determined by the characteristics of the water-bottom or water-top mud interface (roughness, grain size) and not by the characteristics of the underlying sediment volume. That is most likely the reason why 'mud' and 'hard soil' can belong to the same acoustic class. They most likely have similar interface characteristics (smoothness, specular surface).

A lower frequency multibeam (ca. $30 \mathrm{kHz}$ ) would have a larger penetration depth and capture the characteristics of the top sediment volume, and most likely be able to better distinguish between grain-sizes, e.g. muddy sand and sandy mud.

\section{Sampling strategy}

In case of supervised classification, a more intensive calibration sediment sampling campaign is suggested. By taken more samples statistically, the number of outlyers within a certain lithological classe will reduce. The sampling should also be performed over a more structured grid with samples at regular distances along transects crossing the boundaries of previously defined acoustic classes.

A detailed description of the samples on board is crucial. E.g. the presence of a fine mud layer at the top can strongly influence the acoustic backscatter signature, but will not appear from lab analyses. Also the presence of gravel, present as scattered boulders or as a continuous layer will give a very 
different acoustic reflection, although the percentage of boulders determined in the lab will be the same.

In contrast to the supervised classification, in which is started from a number of predefined lithological classes, the unsupervised classification allows to examine purposively why a certain area belongs to a specific acoustic class and the class next to it differs from it, taking into account not only grain size, but also structural elements and mixture of gravel, plant fragments, etc. It was observed during this project that 'hard soil' can belong to different acoustic classes and also 'mud' can have different acoustic signatures (e.g. consolidated crumbly mud versus sludgy mud).

It is recommended to take sediment samples along transects crossing different acoustic classes, in order to capture the lithological and structural evolution over the boundaries. Both the detailed description on board and the lab tests afterwards will show whether the acoustic change is due changing shell content, changing grain size, presence of gravel or plant material, presence of mud layer, etc. Without presuming that the acoustic classes are defined by grain size only (as was the case for the supervised classification).

\section{CONCLUSION}

As the study area is a tide-dominated estuary, water level, currents, salinity and sediment concentration in the water column vary strongly over a tidal cycle. Several tests showed, however, that the moment at which the multibeam data were recorded was not of importance. There were no meaningful differences observed between acoustic maps created from recordings at different moments in the tidal cycle. Variations in salinity, temperature and water depth are correctly compensated by the QTC MULTIVIEW ${ }^{\mathrm{TM}}$ software.

Preference is given to unsupervised classification of the multibeam backscatter data. In a heterogeneous environment as the Lower Sea Scheldt it is important that the entire backscatter data set is used to created acoustic classification maps, instead of applying a catalogue from a restricted reference area to the entire investigation area (supervised classification). The unsupervised classification method should be combined with a structured and detailed sampling campaign which describes more than grain size alone. The obtained optimum number of acoustic classes should be ground-truthed in terms of characteristics of the water-bottom interface when applying a highfrequency multibeam sonar ( $300 \mathrm{kHz}$ ). In case of applying a lower frequency multibeam sonar $(30 \mathrm{kHz})$, the characteristics (especially grain-size) of the underlying sediment volume will become more important.

\section{ACKNOWLEDGEMENTS}

We want to thank the Flemish Hydrography for the high-quality multibeam recordings and for placing survey vessels and crew at our disposal during sampling campaigns.

We also want to acknowledge the Hydrology and Hydraulic Engineering department of VUB (Vrije Universiteit Brussel) and the Flanders Hydraulics Research centre for the analyses of sediment samples. 